\title{
Contabilidade de Custos nas Universidades Brasileiras: uma análise dos planos de ensino nos cursos de Administração
}

\begin{abstract}
Diego Sampaio Vasconcelos Ramalho Lima
Mestrado em andamento em Administração e Controladoria pela Universidade Federal do Ceará - UFC Avenida da Universidade, 2853. Benfica. Fortaleza/CE. CEP: 60020-180 E-mail:diegolima_sampaio@hotmail.com

Maria Naiula Monteiro Pessoa Doutorado em Engenharia de Produção pela Universidade Federal de Santa Catarina UFSC

Professora da Universidade Federal do Ceará - UFC Avenida da Universidade, 2853. Benfica. Fortaleza/CE. CEP: 60020-180 E-mail: naiula@ufc.br

Sandra Maria dos Santos Doutorado em Economia pela Universidade Federal de Pernambuco - UFPE Professora da Universidade Federal do Ceará - UFC Avenida da Universidade, 2853. Benfica. Fortaleza/CE. CEP: 60020-180 E-mail:smsantos@ufc.br
\end{abstract}

Augusto Cézar de Aquino Cabral Doutorado em Administração pela Universidade Federal de Minas Gerais - UFMG Professor da Universidade Federal do Ceará - UFC Avenida da Universidade, 2853. Benfica. Fortaleza/CE. CEP: 60020-180 E-mail:cabral@ufc.br

Clayton Robson Moreira da Silva Mestrado em andamento em Administração e Controladoria pela Universidade Federal do Ceará - UFC Avenida da Universidade, 2853. Benfica. Fortaleza/CE. CEP: 60020-180 E-mail: claytonrmsilva@gmail.com

\section{RESUMO}

O presente estudo tem como objetivo analisar o perfil da disciplina de Contabilidade de Custos nos cursos de Administração das universidades brasileiras, a fim de identificar a estrutura, nomenclatura, metodologias, processo de avaliação, conteúdo programático e bibliografia, bem como comparar as semelhanças e particularidades dos resultados aqui alcançados com os obtidos em estudos anteriores. A pesquisa é de natureza quantitativa e caracteriza-se como descritiva e documental. $O$ universo da pesquisa foi definido com base no Ranking Universitário, Folha 2015, e foi composto pelas 50 melhores universidades brasileiras. De acordo com os resultados obtidos, observa-se 
Contabilidade de Custos nas Universidades Brasileiras: uma análise dos planos de ensino nos cursos de Administração

Diego Sampaio Vasconcelos Ramalho Lima, Maria Naiula Monteiro Pessoa, Sandra Maria dos Santos, Augusto Cézar de Aquino Cabral, Clayton Robson Moreira da Silva

que: (i) há certa homogeneidade na estrutura dos planos de ensino das universidades brasileiras; (ii) a metodologia de ensino mais utilizada é a "Aula Expositiva"; (iii) o processo de avaliação mais comum é a "Prova". A comparação com trabalhos anteriores mostra que cada um possui suas peculiaridades, não se podendo dizer que há plena similaridade entre os resultados aqui obtidos e os resultantes da pesquisa realizada em instituições americanas e o estudo feito em universidades de Santa Catarina. No entanto, pode-se afirmar existir maior proximidade com os resultados do estudo catarinense.

Palavras-chave: Educação. Universidades Brasileiras. Graduação em Administração. Custos.

\section{Cost Accounting in Brazilian Universities: an analysis on management programs}

\section{ABSTRACT}

This study aims to analyse the profile of the discipline of cost accounting in management programs of Brazilian universities in order to identify the structure, name, methodology, evaluation process, content and bibliography, as well as to compare the similarities and peculiarities of the results of this study with the results reached in previous studies. This is a quantitative research, and it is caracterized as descriptive and documental. The universe of the research was defined based on the "Raking Universitário Folha 2015" and it consists of the 50 best Brazilian universities. According to the results, it can be observed that: (i) there is certain homogeneity in the structure of the lesson plans of the Brazilian universities; (ii) the most used methodology is "lecture class"; (iii) the most common evaluation process is "test". Comparing to previous studies, it shows that each one has its peculiarities, not being possible to say that there is full similarity between the results obtained in this study and the results obtained in the research held in American institutions or with the study held in universities from Santa Catarina. However, it is possible to claim that there is more similarity with the study held in Santa Catarina.

Keywords: Education. Brazilian Universities. Management Program. Cost.

\section{INTRODUÇÃO}

As constantes transformações que vêm ocorrendo em um mundo cada vez mais globalizado têm demandado mais preparo dos gestores organizacionais. Os desafios demandam interdisciplinaridade no tratamento de questões a serem resolvidas. Nesse contexto, no campo da gestão, o conhecimento e domínio da Contabilidade são cada 
Contabilidade de Custos nas Universidades Brasileiras: uma análise dos planos de ensino nos cursos de Administração

Diego Sampaio Vasconcelos Ramalho Lima, Maria Naiula Monteiro Pessoa, Sandra Maria dos Santos, Augusto Cézar de Aquino Cabral, Clayton Robson Moreira da Silva

vez mais importantes para a formação de profissionais que estejam aptos ao ingresso no mercado (HART e WANG, 2016).

Essa realidade afeta diretamente o ensino superior de graduação (HOPPER, 2012), tanto na teoria como na prática, o que torna necessário o processo de reavaliação de cada disciplina no currículo estabelecido para a formação de um determinado profissional (GODOY et al., 2004).

Entendendo a relevância do conhecimento contábil na gestão empresarial, Raupp et al. (2009) afirmam que os cursos de Bacharelado em Administração devem estruturar as disciplinas de Contabilidade de maneira que possam inserir no processo ensino-aprendizagem os conhecimentos e discussões contábeis necessários ao desempenho profissional do administrador.

O ensino de Contabilidade para não contadores, conforme Tcheou (2002), tem sido pouco explorado em artigos, seminários e congressos, apesar de a Contabilidade ser contemplada como disciplina não só nos currículos das Faculdades de Administração, como também nas de Direito, Engenharia e Economia. A inserção acontece também nos cursos de educação continuada, como cursos de especialização, e nos programas de atualização e reciclagem em Contabilidade.

Assim, os estudos que analisam a grade curricular de um curso ou determinada disciplina são relevantes. Alguns pensadores acreditam que as instituições de ensino superior devem adaptar-se às exigências do mercado profissional, buscando um currículo compatível e capaz de contribuir para a formação do profissional. Outros entendem que o Ensino Superior deveria estar à frente de questões profissionais (SPLITTER et al., 2014).

No âmbito da Contabilidade, insere-se a Contabilidade de Custos. Para Megliorini (2012), conhecer custos é uma condição essencial para gerir uma empresa, independentemente do tipo e do porte. Por isso, não se pode deixar o cálculo dos custos a um plano secundário, pois eles constituem ferramentas auxiliares da boa administração (KONT, 2013). 
Contabilidade de Custos nas Universidades Brasileiras: uma análise dos planos de ensino nos cursos de Administração

Diego Sampaio Vasconcelos Ramalho Lima, Maria Naiula Monteiro Pessoa, Sandra Maria dos Santos, Augusto Cézar de Aquino Cabral, Clayton Robson Moreira da Silva

Diante dessa exposição, o presente estudo tem a seguinte questão de pesquisa: Qual o perfil da disciplina de custos no ensino de graduação em Administração das universidades brasileiras?

Esta pesquisa tem como objetivo geral analisar o perfil da disciplina de custos no ensino de graduação em Administração nas universidades brasileiras, por meio da identificação de elementos de ensino e aplicação didática. Para o alcance deste objetivo, foram definidos os seguintes objetivos específicos: (1) identificar as denominações utilizadas para caracterizar as disciplinas que possuem o conteúdo de custos; (2) analisar a estrutura dos planos de ensino das disciplinas, os conteúdos programáticos e as bibliografias; (3) verificar as metodologias de ensino e avaliativas utilizadas; (4) comparar as semelhanças e particularidades dos resultados alcançados neste estudo com os obtidos nos estudos de Splitter et al. (2014), Raupp et al. (2009a) e Raupp et al. (2009b).

O estudo é de natureza quantitativa e caracteriza-se como uma pesquisa documental e descritiva. É uma replicação do trabalho desenvolvido por Sombra et al. (2016), que teve como foco os cursos de graduação em Contabilidade. Ao mesmo tempo, a presente pesquisa busca comparar os resultados obtidos com os resultados de estudos com foco na Administração.

\section{REVISÃO DA LITERATURA}

\subsection{Contabilidade de Custos nos Cursos de Administração}

Estudos diversos (COVRE, 1991; LOPES, 2002; NICOLINI, 2003) descrevem o início dos cursos de ensino superior na área de Administração no Brasil por volta de 1950, a partir da demanda por profissionais capacitados, gerada pelas empresas multinacionais, escritórios de consultoria e pelo próprio governo.

O crescimento de empresas nacionais e multinacionais intensificou a procura por profissionais aptos na tomada de decisão organizacional. Os aspectos mais enfatizados na formação dos administradores eram o conhecimento e habilidades técnicas mais 
Contabilidade de Custos nas Universidades Brasileiras: uma análise dos planos de ensino nos cursos de Administração

Diego Sampaio Vasconcelos Ramalho Lima, Maria Naiula Monteiro Pessoa, Sandra Maria dos Santos, Augusto Cézar de Aquino Cabral, Clayton Robson Moreira da Silva

apropriadas para a execução das atividades impostas pelas organizações de negócios. As décadas de 1960 e 1970 presenciaram uma rápida expansão do ensino superior em Administração, incentivada pelos mesmos fatores de implantação dos cursos (CANOPF et al., 2005). Nessa esteira, observa-se que há necessidade do domínio de assuntos técnicos específicos, de caráter interdisciplinar, para dar o respaldo necessário na tomada de decisão do gestor. Dentre esses assuntos, aponta-se o domínio da informação contábil como forte aliado no processo decisório para profissionais da administração (XAVIER FILHO et al., 2015).

A Contabilidade de Custos foi um resultado do processo de industrialização, resultante da Revolução Industrial (século XVIII). Até então, existia basicamente a Contabilidade Financeira que, desenvolvida na Era Mercantilista, estava bem estruturada para atender às empresas comerciais. Com o surgimento da indústria, tornou-se bastante complexa a tarefa de avaliar os estoques dos produtos existentes e produzidos na empresa, pelo fato de que os produtos incorporam diversos fatores de produção utilizados para sua obtenção. Assim, a preocupação inicial era a de tornar a Contabilidade de Custos uma forma de resolver os problemas de mensuração monetária dos estoques e do resultado, não a de fazer dela um instrumento gerencial (KONT, 2013; CREPALDI, 2010).

Crepaldi (2010) acrescenta ainda que, nas últimas décadas, a Contabilidade de Custos vem evoluindo e se modernizando, deixando de ser mera auxiliar na avaliação de estoques e de lucros globais, para tornar-se um importante instrumento de controle e suporte às tomadas de decisões.

Em um mercado altamente competitivo, o conhecimento acerca da administração é um fator determinante para o bom desempenho de uma organização. O entendimento dos custos é uma condição essencial para gerir uma empresa, independentemente do tipo e do porte. Por isso, não se pode deixar o cálculo dos custos a um plano secundário, pois eles constituem ferramentas auxiliares da boa administração (MEGLLIORINI, 2012). 
Contabilidade de Custos nas Universidades Brasileiras: uma análise dos planos de ensino nos cursos de Administração

Diego Sampaio Vasconcelos Ramalho Lima, Maria Naiula Monteiro Pessoa, Sandra Maria dos Santos, Augusto Cézar de Aquino Cabral, Clayton Robson Moreira da Silva

A disciplina de Contabilidade é considerada obrigatória nos cursos de graduação em Administração. As Diretrizes Curriculares Nacionais do Curso de Graduação em Administração, bacharelado, instituídas por meio da Resolução ํㅜ 4, de 13 de julho de 2005, estabelecem a obrigatoriedade dessa disciplina na composição das matrizes curriculares.

A organização das disciplinas cabe a cada Instituição de Ensino. Segundo Harada (2005), pela própria natureza do curso de Administração de Empresas, a carga horária das disciplinas da área de Contabilidade pode ser insuficiente para repassar todos os fundamentos de Contabilidade.

\subsection{Planejamento e plano de ensino}

O planejamento de ensino constitui-se como um elemento de manifestação dos princípios utilizados pelo professor em sala de aula. Esses princípios conduzem os alunos com a finalidade de alcançar os objetivos educacionais determinados (PILETTI, 2010). Para Leal (2005), o planejamento do ensino tem características que the são próprias, isto porque lida com os sujeitos aprendentes, portanto sujeitos em processo de formação humana. Para tal empreendimento, o professor realiza passos que se complementam e se interpenetram na ação didático-pedagógica.

Gil (2011) afirma que o planejamento do ensino está alicerçado no planejamento curricular e visa ao direcionamento sistemático das atividades a serem desenvolvidas dentro e fora de sala de aula, facilitando o aprendizado dos estudantes. O autor afirma que o planejamento é elaborado somente pelo professor responsável pela disciplina, mas recomenda que mais professores compartilhem a responsabilidade de sua elaboração.

De acordo com Padilha (2001), o plano é a apresentação sistematizada e justificada das decisões tomadas, relativas à ação a realizar. Assim, como afirmam Splitter et al. (2014), plano de ensino pode ser considerado o planejamento e organização das aulas que permitam aos alunos entenderem com clareza o que será 
Contabilidade de Custos nas Universidades Brasileiras: uma análise dos planos de ensino nos cursos de Administração

Diego Sampaio Vasconcelos Ramalho Lima, Maria Naiula Monteiro Pessoa, Sandra Maria dos Santos, Augusto Cézar de Aquino Cabral, Clayton Robson Moreira da Silva

abordado com relação ao tema discutido na disciplina em determinado período objetivando uma formação de qualidade.

Para a estruturação de um plano de ensino, é necessária a presença dos seguintes elementos: determinação dos objetivos; seleção e organização dos conteúdos; seleção e organização dos procedimentos de ensino; seleção de recursos e seleção de procedimentos de avaliação (PILETTI, 2010).

Os objetivos caracterizam-se como a descrição clara e direta do que se busca atingir por meio das atividades que serão desenvolvidas (PILETTI, 2010). O conteúdo deve relacionar-se intimamente com os objetivos estabelecidos, buscando, dessa forma, alcançá-los (GIL, 2011). A ementa é um resumo dos conteúdos que irão ser trabalhados no projeto da disciplina (LEAL, 2005).

A bibliografia apresenta as sugestões de literatura que deverão ser feitas para aprender os conteúdos. As estratégias de ensino exibem quais formas de ensino foram escolhidas para facilitar a aprendizagem do conteúdo. Podem ser, por exemplo, seminários, aulas expositivas, estudos de caso (GIL, 2011).

Os recursos de ensino são os elementos utilizados no ambiente da aprendizagem, como o professor, quadro, giz, bibliotecas (PILETTI, 2010). O cronograma é a apresentação da distribuição das atividades durante o período letivo (GIL, 2011).

Por fim, o método de avaliação tem como finalidade determinar o grau dos resultados alcançados, relacionando-os com os objetivos definidos no plano de ensino (PILETTI, 2010). Elas podem acontecer de forma oral, escrita, dissertativa, objetiva (GIL, 2011).

\subsection{Trabalhos anteriores sobre o ensino de disciplinas na área de Contabilidade}

Visando embasar o presente estudo, foi realizada uma revisão na literatura nacional e internacional. Dentre os trabalhos identificados, destacam-se os estudos de Splitter et al. (2014), Raupp et al. (2009a) e Raupp et al. (2009b). 
Contabilidade de Custos nas Universidades Brasileiras: uma análise dos planos de ensino nos cursos de Administração

Diego Sampaio Vasconcelos Ramalho Lima, Maria Naiula Monteiro Pessoa, Sandra Maria dos Santos, Augusto Cézar de Aquino Cabral, Clayton Robson Moreira da Silva

O estudo de Splitter et al. (2014) tem como objetivo investigar, através de pesquisa científica, os planos de ensino das disciplinas que abordam custos nos cursos de graduação em Administração e Negócios nas universidades norte-americanas. Utilizou-se a pesquisa documental e a amostragem por acessibilidade e, assim, sua amostra é composta por 11 (onze) universidades norteamericanas qualificadas pelo ranking da Revista Business Week e 16 (dezesseis) planos de ensino. Dentre seus resultados, pode-se destacar que, durante o desenvolvimento da disciplina de contabilidade de custos, há grande pluralidade de conteúdos abordados; as metodologias de ensino mais utilizadas foram aulas expositivas e atividades de casa; os processos de avaliação mais comuns abordados são provas, participação/presença; e quanto às referências, não foi detectada grande diversidade, havendo menção de apenas oito obras.

O estudo de Raupp et al. (2009a) tem como objetivo apresentar o perfil do ensino de Contabilidade Geral e de Custos nos cursos de graduação em Administração do Estado de Santa Catarina por meio dos elementos constantes nos planos de ensino, enquanto o estudo de Raupp et al. (2009b) tem foco apenas a disciplina de Contabilidade de Custos.

Nessa esteira, ambos os estudos, Raupp et al. (2009a) e Raupp et al. (2009b) utilizaram-se de amostragem por acessibilidade, alcançando 28 Instituições de Ensino Superior (IES), perfazendo $34,60 \%$ da população. Dentre os resultados para a disciplina de Contabilidade de Custos, pode-se destacar que, durante o desenvolvimento da disciplina, há grande pluralidade de conteúdos abordados: as metodologias de ensino mais utilizadas foram aulas expositivas e exercícios; os processos de avaliação mais comuns abordados são provas e trabalhos; e quanto às referências, foi detectada grande diversidade, porém com forte predominância do livro "Contabilidade de Custos", de Eliseu Martins.

Ressalta-se também o estudo desenvolvido por Sombra et al. (2016), que foi a base para a presente pesquisa. Nele, os autores objetivam identificar o perfil das disciplinas de contabilidade de custos nos cursos de graduação em Ciências Contábeis 
Contabilidade de Custos nas Universidades Brasileiras: uma análise dos planos de ensino nos

cursos de Administração

Diego Sampaio Vasconcelos Ramalho Lima, Maria Naiula Monteiro Pessoa, Sandra Maria dos Santos, Augusto Cézar de Aquino Cabral, Clayton Robson Moreira da Silva

nas universidades brasileiras. Caracteriza-se como uma pesquisa descritiva, de natureza quantitativa e do tipo documental. Foram analisadas 18 universidades, entre as 50 primeiras classificadas pelo Ranking Universitário Folha 2014, e 24 planos de ensino. Alguns resultados foram: uniformidade entre os elementos que compõem os planos de ensino. Dentre os principais conteúdos, destaca-se: "Introdução à contabilidade de custos". O método de ensino mais citado foram as "Aulas expositivas"; a forma avaliativa mais usual é a "Prova"; o livro "Contabilidade de Custos", do autor Eliseu Martins, foi o mais mencionado. Tal estudo também faz uma análise comparativa com o estudo de Splitter et al. (2014).

Portanto, percebe-se que existe uma gama de trabalhos que abordam diversos aspectos do ensino de disciplinas na área da Contabilidade, tanto nos cursos de Administração como nos de Ciências Contábeis. Todos buscam contribuir, de alguma forma, para a melhoria do ensino das diversas disciplinas. Contudo, dado o foco da presente pesquisa, tomou-se como base os estudos de Splitter et al. (2014), Raupp et al. (2009a), Raupp et al. (2009b) e Sombra et al. (2016).

\section{METODOLOGIA}

Quanto à natureza do problema, o estudo utilizou-se da abordagem quantitativa. A abordagem quantitativa caracteriza-se pelo emprego de técnicas estatísticas, desde as mais simples, como percentual, média, desvio-padrão, às mais complexas, como coeficientes de correlação e análise de regressão (RICHARDSON, 2008).

Quanto aos objetivos, essa pesquisa consiste em um estudo do tipo descritivo. A pesquisa descritiva preocupa-se em observar os fatos, registrá-los, analisá-los, classificá-los e interpretá-los, não havendo interferência do pesquisador (ANDRADE, 2008). O caráter descritivo embasa-se na intenção da pesquisa de descrever situações e fornecer informações contextuais que poderão servir de base para pesquisas explicativas mais aprofundadas (SAMPIERI et al., 2013; DESLAURIERS e KÉRISIT, 2008). 
Contabilidade de Custos nas Universidades Brasileiras: uma análise dos planos de ensino nos cursos de Administração

Diego Sampaio Vasconcelos Ramalho Lima, Maria Naiula Monteiro Pessoa, Sandra Maria dos Santos, Augusto Cézar de Aquino Cabral, Clayton Robson Moreira da Silva

No tocante aos procedimentos técnicos, a pesquisa é do tipo documental. A pesquisa documental tipifica-se por utilizar documentos elaborados com finalidades diversas como, por exemplo, documentos internos de uma organização (GIL, 2011).

Os documentos utilizados foram os planos de ensino das universidades brasileiras, que foram analisados a fim de se encontrar os resultados da pesquisa.

A princípio, as 50 melhores universidades brasileiras classificadas pelo Ranking Universitário Folha (RUF) 2015 foram escolhidas como universo de estudo. Se o percentual de resposta fosse muito baixo, partir-se-ia para um aumento da população de pesquisa.

O RUF é uma avaliação anual do ensino superior do Brasil feito pela Folha de São Paulo desde 2012. No ranking das universidades, estão todas as universidades brasileiras, públicas e privadas. São cinco os indicadores considerados para a elaboração do ranking: pesquisa, internacionalização, inovação, ensino e mercado. E os dados que compõem esses indicadores são coletados por uma equipe da Folha em bases de patentes brasileiras, em bases de periódicos científicos, em bases do MEC e em pesquisas nacionais de opinião feitas pelo Datafolha (RUF - Folha de São Paulo, 2015).

O RUF também possui a classificação de acordo com os cursos de graduação. No entanto, utilizou-se na pesquisa a classificação geral, que analisa todos os cursos de graduação de cada instituição.

A taxa de respostas e recebimento de documentos (planos de ensino) foi igual a $60 \%$, ou seja, a amostra da pesquisa é composta por 30 universidades. Desse total, 26 instituições enviaram um plano de ensino e 4 enviaram dois planos de ensino, totalizando 34 planos de ensino. A Tabela 1 apresenta as universidades que constituem a amostra em estudo. 
Contabilidade de Custos nas Universidades Brasileiras: uma análise dos planos de ensino nos cursos de Administração Diego Sampaio Vasconcelos Ramalho Lima, Maria Naiula Monteiro Pessoa, Sandra Maria dos Santos, Augusto Cézar de Aquino Cabral, Clayton Robson Moreira da Silva

Tabela 1: Amostra da pesquisa

\begin{tabular}{|c|c|c|c|c|c|c|c|}
\hline № & Universidade & $\begin{array}{c}\text { Posição } \\
\text { Ranking }\end{array}$ & № planos & № & Universidade & $\begin{array}{c}\text { Posição } \\
\text { Ranking }\end{array}$ & № planos \\
\hline 1 & USP & $1^{\circ}$ & 1 & 16 & UEM & $24^{\circ}$ & 2 \\
\hline 2 & UFRJ & $2^{\circ}$ & 1 & 17 & UFRN & $25^{\circ}$ & 1 \\
\hline 3 & UFMG & $3^{\circ}$ & 1 & 18 & UFU & $26^{\circ}$ & 1 \\
\hline 4 & UNICAMP & $4^{\circ}$ & 2 & 19 & UFPEL & $29^{\circ}$ & 1 \\
\hline 5 & UFRGS & $5^{\circ}$ & 1 & 20 & UFES & $30^{\circ}$ & 1 \\
\hline 6 & UFSC & $7^{\circ}$ & 1 & 21 & PUC-PR & $31^{\circ}$ & 1 \\
\hline 7 & UFC & $11^{\circ}$ & 1 & 22 & MACKENZIE & $32^{\circ}$ & 1 \\
\hline 8 & UFSCar & $12^{\circ}$ & 1 & 23 & UFMT & $33^{\circ}$ & 1 \\
\hline 9 & UFF & $13^{\circ}$ & 1 & 24 & UFMS & $36^{\circ}$ & 1 \\
\hline 10 & UFV & $16^{\circ}$ & 2 & 25 & UFCG & $38^{\circ}$ & 2 \\
\hline 11 & UFG & $17^{\circ}$ & 1 & 26 & UDESC & $41^{\circ}$ & 1 \\
\hline 12 & UFSM & $18^{\circ}$ & 1 & 27 & UFTPR & $46^{\circ}$ & 1 \\
\hline 13 & PUC-RJ & $19^{\circ}$ & 1 & 28 & PUC-MG & $47^{\circ}$ & 1 \\
\hline 14 & PUC-RS & $20^{\circ}$ & 1 & 29 & UFAM & $48^{\circ}$ & 1 \\
\hline 15 & UNIFESP & $22^{\circ}$ & 1 & 30 & UFRRJ & $49^{\circ}$ & 1 \\
\hline
\end{tabular}

Fonte: Elaborado pelos autores.

A pesquisa se utilizou da documentação indireta como técnica de coleta de dados. Para Marconi e Lakatos (2008), a coleta de dados baseada em documentação indireta é a fase da pesquisa realizada com o intuito de recolher informações prévias sobre o campo de interesse.

A coleta de dados foi feita por meio dos e-mails e dos documentos diretamente selecionados nos sites institucionais das universidades de ensino. A coleta começou pela busca nos sites das 50 Universidades. O primeiro procedimento era verificar se a universidade possuía o curso de graduação em Administração. Apenas uma instituição não possuía o curso de Administração: a Universidade Federal do ABC (UFABC).

Para as outras 49 Universidades, foram feitas pesquisas nos sites institucionais. Primeiramente, procurou-se o plano de ensino da disciplina de custos dos cursos de Administração. Oito instituições divulgam esse documento diretamente no site. Para 
Contabilidade de Custos nas Universidades Brasileiras: uma análise dos planos de ensino nos cursos de Administração

Diego Sampaio Vasconcelos Ramalho Lima, Maria Naiula Monteiro Pessoa, Sandra Maria dos Santos, Augusto Cézar de Aquino Cabral, Clayton Robson Moreira da Silva

aquelas que não possuíam, coletou-se o e-mail do coordenador do curso de administração a fim de enviar solicitação formal do plano de ensino da disciplina em estudo.

Os planos de ensino foram analisados individualmente, e os dados foram tabulados de acordo com cada objetivo específico da pesquisa. Também foram realizados cálculos dos percentuais representativos de cada item, a fim de facilitar a análise. Fez-se uso de planilhas Excel para tabulação.

Posteriormente, confrontaram-se os resultados da pesquisa com os resultados dos estudos de Splitter et al. (2014), Raupp et al. (2009a) e Raupp et al. (2009b), a fim de obter uma comparação entre os estudos.

\section{APRESENTAÇÃo E ANÁLISE DOS RESULTADOS}

\subsection{Denominações e cargas horárias das disciplinas de Custos}

Com base nos planos de ensino obtidos, primeiramente observaram-se as disciplinas que oferecem o conteúdo de Contabilidade de Custos para, então, a partir da análise dos 34 planos de ensino, definir quais são as denominações que a disciplina recebe em cada universidade, bem como as cargas horárias aplicadas em cada uma delas. As nomenclaturas mais utilizadas foram: "Contabilidade de Custos", "Contabilidade Gerencial" e "Gestão de Custos", todas com cinco menções, representando quase $15 \%$ da amostra cada uma. Logo em seguida, aparece "Administração de Custos", com 4 menções. As demais menções tendem a mesclar as nomenclaturas mais citadas, como: "Administração de Custos Gerenciais" e "Gestão Estratégica de Custos", e apresentaram, no máximo, duas menções. A Tabela 2 mostra as nomenclaturas encontradas. 
Contabilidade de Custos nas Universidades Brasileiras: uma análise dos planos de ensino nos cursos de Administração

Diego Sampaio Vasconcelos Ramalho Lima, Maria Naiula Monteiro Pessoa, Sandra Maria dos Santos, Augusto Cézar de Aquino Cabral, Clayton Robson Moreira da Silva

Tabela 2: Nomenclaturas e cargas horárias das disciplinas.

\begin{tabular}{|c|c|c|c|c|}
\hline Nomenclatura & Menções & IES & $\mathrm{C} / \mathrm{H}$ & $\%$ \\
\hline \multirow{5}{*}{ Contabilidade de Custos } & \multirow{5}{*}{5} & UFC & $64 \mathrm{~h}$ & \multirow{5}{*}{14,71} \\
\hline & & USP & $60 \mathrm{~h}$ & \\
\hline & & PUC-RS & $60 \mathrm{~h}$ & \\
\hline & & UDESC & $72 \mathrm{~h}$ & \\
\hline & & UFCG & $60 \mathrm{~h}$ & \\
\hline \multirow{5}{*}{ Contabilidade Gerencial } & \multirow{5}{*}{5} & UFG & $64 \mathrm{~h}$ & \multirow{5}{*}{14,71} \\
\hline & & MACKENZIE & 4h/semana & \\
\hline & & UFRRJ & $60 \mathrm{H}$ & \\
\hline & & UFRJ & $60 \mathrm{~h}$ & \\
\hline & & UFCG & $60 \mathrm{~h}$ & \\
\hline \multirow{5}{*}{ Gestão de Custos } & \multirow{5}{*}{5} & PUC-MG & $68 \mathrm{~h}$ & \multirow{5}{*}{14,71} \\
\hline & & UFMG & $60 \mathrm{~h}$ & \\
\hline & & UFTPR & $54 \mathrm{~h}$ & \\
\hline & & UFAM & $60 \mathrm{~h}$ & \\
\hline & & UFSCar & $60 \mathrm{~h}$ & \\
\hline \multirow{4}{*}{ Administração de Custos } & \multirow{4}{*}{4} & UFSC & $72 \mathrm{~h}$ & \multirow{4}{*}{11,76} \\
\hline & & UFU & $60 \mathrm{~h}$ & \\
\hline & & UFPEL & $68 \mathrm{~h}$ & \\
\hline & & UFES & $60 \mathrm{~h}$ & \\
\hline \multirow{2}{*}{ Custos Empresariais } & \multirow{2}{*}{2} & UNIFESP & $60 \mathrm{~h}$ & \multirow{2}{*}{5,88} \\
\hline & & UFMT & $64 \mathrm{~h}$ & \\
\hline \multirow{2}{*}{ Contabilidade Gerencial e de Custos } & \multirow{2}{*}{2} & PUC-RJ & - & \multirow{2}{*}{5,88} \\
\hline & & UFMS & - & \\
\hline Gestão Estratégica de Custos & 1 & PUC-PR & $80 \mathrm{~h}$ & 2,94 \\
\hline Administração de Custos Gerenciais & 1 & UFRN & $60 \mathrm{~h}$ & 2,94 \\
\hline Custos & 1 & UFSM & $60 \mathrm{~h}$ & 2,94 \\
\hline Custos I & 1 & UEM & $68 \mathrm{~h}$ & 2,94 \\
\hline Custos II & 1 & UEM & $68 \mathrm{~h}$ & 2,94 \\
\hline Fundamentos de Finanças e Custos & 1 & UNICAMP & $60 \mathrm{~h}$ & 2,94 \\
\hline Metodologia Básica de Custos & 1 & UFRGS & $60 \mathrm{~h}$ & 2,94 \\
\hline Análise de Custos & 1 & UNICAMP & $30 \mathrm{~h}$ & 2,94 \\
\hline Análise de Custos I & 1 & UFV & $60 \mathrm{~h}$ & 2,94 \\
\hline Análise de Custos II & 1 & UFV & $60 \mathrm{~h}$ & 2,94 \\
\hline Controladoria & 1 & UFF & $60 \mathrm{~h}$ & 2,94 \\
\hline
\end{tabular}


Contabilidade de Custos nas Universidades Brasileiras: uma análise dos planos de ensino nos cursos de Administração Diego Sampaio Vasconcelos Ramalho Lima, Maria Naiula Monteiro Pessoa, Sandra Maria dos Santos, Augusto Cézar de Aquino Cabral, Clayton Robson Moreira da Silva

TOTAL 34

$-$ 100

Fonte: Elaborado pelos autores.

Percebe-se, portanto, que não há uma homogeneidade quanto à nomenclatura que define a disciplina que aborda o conteúdo de custos nos cursos de graduação em Administração das universidades brasileiras.

No que tange às cargas horárias presentes nos planos de ensino, observa-se que a quantidade de 60h/a é a mais frequente, estando presente em 19 planos de ensino. Além disso, pode-se observar que a carga horária mais extensa é de $80 \mathrm{~h} / \mathrm{a}$, sendo referente ao plano de ensino do curso de Administração da PUC-PR, o que indica uma maior profundidade em relação à abordagem de custos nesse plano de ensino. As IES UEM, UFCG, UFV e UNICAMP contam com mais de um plano de ensino que contempla a temática Custos, indicando maior robustez no ensino de custos nos cursos de Administração dessas IES. Os planos de ensino da PUC-RJ e UFMS não apresentam a carga horária despendida.

Os resultados referentes às nomenclaturas aproximam-se dos encontrados por Raupp et al. (2009a) e Raupp et al. (2009b). No entanto, difere do estudo de Splitter et al. (2014). Os primeiros autores concluíram que nem todas as Instituições analisadas possuem uma disciplina específica para o conteúdo de Contabilidade de Custos. As denominações mais utilizadas foram "Administração de Custos", "Custos Empresarias" e "Gestão de Custos", cada uma com 15\% de menção. Posteriormente, encontram-se "Contabilidade Gerencial", "Custos" e "Contabilidade de Custos".

Já no estudo nas universidades norteamericanas de Splitter et al. (2014), o resultado encontrado foi um domínio da nomenclatura "Managerial Accouting" ou "Contabilidade Gerencial", com 56\% de menção. Percebe-se, consequentemente, uma homogeneidade na nomenclatura da disciplina que aborda o conteúdo de custos nas universidades estrangeiras.

Assim, os resultados, no que tange à nomenclatura do presente estudo feito nas universidades brasileiras, se aproximam dos resultados dos estudos feitos nas 
Contabilidade de Custos nas Universidades Brasileiras: uma análise dos planos de ensino nos cursos de Administração

Diego Sampaio Vasconcelos Ramalho Lima, Maria Naiula Monteiro Pessoa, Sandra Maria dos Santos, Augusto Cézar de Aquino Cabral, Clayton Robson Moreira da Silva

instituições do Estado de Santa Catarina, porém diferem dos resultados do estudo norteamericano.

\subsection{Estrutura dos planos de ensino}

A análise da estrutura dos planos de ensino das universidades brasileiras mostra que existe uniformidade em 6 dos 12 itens analisados. Os seis primeiros componentes foram mencionados em mais de $82 \%$ dos planos de ensino. Os componentes presentes em todos os 34 planos de ensino são: "Disciplina" e "Bibliografia", como mostra a Tabela 3.

Tabela 3: Estrutura dos planos de ensino

\begin{tabular}{|c|c|c|}
\hline Composição & Menções & $\%$ \\
\hline Disciplina & 34 & 100 \\
\hline Bibliografia & 34 & 100 \\
\hline Ementa & 33 & 97,06 \\
\hline Carga horária & 32 & 94,12 \\
\hline Objetivos & 28 & 82,35 \\
\hline Conteúdo & 28 & 82,35 \\
\hline Avaliação & 14 & 41,17 \\
\hline Semestre & 14 & 41,17 \\
\hline Estratégias de ensino & 13 & 32,35 \\
\hline Recurso de ensino & 10 & 29,41 \\
\hline Nome/Contato do Professor & 10 & 29,41 \\
\hline Cronograma & 3 & 8,82 \\
\hline
\end{tabular}

Fonte: Elaborado pelos autores.

Os dois próximos itens, "Ementa" e "Carga horária", foram mencionados 97\% e $94 \%$, respectivamente, sendo, então, quase unanimidades nos planos de ensino da disciplina de custos. Os itens seguintes foram "Objetivos" e "Conteúdo". Ambos foram mencionados 28 vezes, representando 82\%; portanto, apenas 6 planos de ensino dos 34 participantes da amostra não continham esses tópicos na estrutura. 
Contabilidade de Custos nas Universidades Brasileiras: uma análise dos planos de ensino nos cursos de Administração

Diego Sampaio Vasconcelos Ramalho Lima, Maria Naiula Monteiro Pessoa, Sandra Maria dos Santos, Augusto Cézar de Aquino Cabral, Clayton Robson Moreira da Silva

Os próximos cinco itens apresentaram taxa de menção entre $29 \%$ e $41 \%$, sendo então não muito presentes nos planos de ensino. São eles: "Avaliação" e "Semestre", ambos com 41\% de menção; "Estratégias de ensino", com 32\%; e "Recursos de ensino" e "Nome/Contato do Professor", ambos com $29 \%$ de menção entre os trinta e quatro planos.

O último componente identificado foi "Cronograma". Ele foi mencionado em apenas 3 planos de ensino, o que significa apenas $9 \%$ da amostra em estudo. Portanto, percebe-se que esse componente não costuma ser mencionado na estrutura dos planos.

Os resultados, quando comparados ao estudo de Splitter et al. (2014), apresentam variações significativas. Os resultados dos autores mostram que há certa homogeneidade na estrutura dos planos de ensino nas universidades norte-americanas estudadas, uma vez que os cinco primeiros itens (horário de atendimento extraclasse, método de avaliação, nome e contato do professor, objetivo da disciplina e uso de referências) foram encontrados em todos os planos de ensino da amostra, e os outros itens analisados não tiveram grande disparidade. Percebe-se que o método de avaliação e o contato do professor estão presentes em todos os planos, diferentemente do atual estudo, no qual apenas $41 \%$ e $29 \%$, respectivamente, contêm esses itens.

Outro item que apresentou elevada discrepância foi o cronograma. No estudo de Splitter et al. (2014), 90\% dos planos apresentavam-no na estrutura, enquanto que, no atual estudo, esse item apresentou pouca significância, aparecendo apenas 8\%.

Portanto, percebe-se que, na análise comparativa da estrutura dos planos de ensino, não há homogeneidade entre o atual estudo e o de Splitter et al. (2014). Quanto aos estudos de Raupp et al. (2009a) e Raupp et al. (2009b), não se pôde fazer comparação, pois os autores não apresentaram resultados para o aspecto da estrutura dos planos de ensino. 
Contabilidade de Custos nas Universidades Brasileiras: uma análise dos planos de ensino nos cursos de Administração Diego Sampaio Vasconcelos Ramalho Lima, Maria Naiula Monteiro Pessoa, Sandra Maria dos Santos, Augusto Cézar de Aquino Cabral, Clayton Robson Moreira da Silva

\subsection{Tópicos do conteúdo programático das disciplinas}

Vinte e oito (28) dos 34 planos continham conteúdo programático em sua estrutura. Portanto, esses planos foram analisados e, de acordo com a Tabela 4, percebe-se que os principais tópicos abordados no conteúdo programático foram: "Conceitos e Terminologia de Custos", com 85\%; "Classificação dos custos e despesas" e "Custeio variável", ambos com 82\%; e "Custeio por absorção", com 78\%. Percebe-se que não houve um conteúdo que fosse abordado em todos os planos de ensino. A análise dos tópicos citados nos conteúdos programáticos mostra certa homogeneidade e também uma pluralidade de assuntos abordados.

Outros tópicos explorados são: "Origens da Contabilidade de Custos/Gerencial", com $68 \%$ de menção, e "Ponto de equilíbrio" e "Margem de segurança', ambos com $60 \%$ de menção. O tópico "Relação Custo/Volume/Lucro" não teve uma quantidade tão alta de menções, apenas $42 \%$, ou seja, apenas 12 dos 28 planos analisados. Outros conteúdos abordados foram "Princípios contábeis aplicados a custos", "Análise de custos" e "Gestão estratégica de custos", todos com 14\% de menção; apenas quatro planos mencionavam-nos.

Outros conteúdos foram mencionados, porém em apenas um plano de ensino cada. Por isso, foram inseridos no item "Outros tópicos abordados". Todos os conteúdos são mostrados na Tabela 4. 
Contabilidade de Custos nas Universidades Brasileiras: uma análise dos planos de ensino nos cursos de Administração

Diego Sampaio Vasconcelos Ramalho Lima, Maria Naiula Monteiro Pessoa, Sandra Maria dos Santos, Augusto Cézar de Aquino Cabral, Clayton Robson Moreira da Silva

Tabela 4: Tópicos abordados nos conteúdos programáticos

\begin{tabular}{|c|c|c|}
\hline Tópicos & Menções & $\%$ \\
\hline Conceitos e Terminologia de Custos & 24 & 85,71 \\
\hline Classificação dos custos e despesas & 23 & 82,14 \\
\hline Custeio variável & 23 & 82,14 \\
\hline Custeio por absorção & 22 & 78,57 \\
\hline Origens da Contabilidade de Custos / Gerencial & 19 & 67,86 \\
\hline Ponto de Equilíbrio & 17 & 60,71 \\
\hline Margem de Contribuição & 17 & 60,71 \\
\hline Métodos/Sistemas de custeio & 15 & 53,57 \\
\hline Departamentalização & 13 & 46,43 \\
\hline Custeio por atividades (ABC) & 12 & 42,86 \\
\hline Custos Indiretos de Fabricação/Rateio CIF & 12 & 42,86 \\
\hline Formação do Preço de Venda & 12 & 42,86 \\
\hline Relação Custo/Volume/Lucro & 12 & 42,86 \\
\hline Custo padrão & 10 & 35,71 \\
\hline Alavancagem & 9 & 32,14 \\
\hline Materiais Diretos & 8 & 28,57 \\
\hline Margem de Segurança & 8 & 28,57 \\
\hline Sistema de acumulação de custos & 8 & 28,57 \\
\hline Avaliação de estoques & 7 & 25 \\
\hline Custo de mão de obra & 7 & 25 \\
\hline Centros de Custos & 6 & 21,43 \\
\hline Gestão Estratégica de Custos & 4 & 14,28 \\
\hline Análise dos custos & 4 & 14,28 \\
\hline Princípios contábeis aplicados a custos & 4 & 14,28 \\
\hline Outros tópicos abordados & 10 & - \\
\hline
\end{tabular}

Fonte: Elaborado pelos autores.

No estudo de Splitter et al. (2014), apenas um plano de ensino não aborda o "Custeio por atividades $(A B C)$ ", o que representou $87,50 \%$. O segundo tópico mais 
Contabilidade de Custos nas Universidades Brasileiras: uma análise dos planos de ensino nos cursos de Administração

Diego Sampaio Vasconcelos Ramalho Lima, Maria Naiula Monteiro Pessoa, Sandra Maria dos Santos, Augusto Cézar de Aquino Cabral, Clayton Robson Moreira da Silva

citado foi "Tomada de decisão", com 81\%, seguido de "Orçamento", Relação Custo/Volume/Lucro" e "Conceitos e terminologia de custos", todos acima de 68\%.

Nos estudos de Raupp et al. (2009a) e Raupp et al. (2009b), a "Relação Custo/Volume/Lucro" é o item mais citado. Em seguida, com igual ocorrência, cita-se o "Custeio variável" e o "Ponto de equilíbrio". A "Margem de contribuição" ocupa a terceira colocação. Alguns dos conteúdos menos citados correspondem aos "Conceitos e terminologia de custos".

A análise comparativa entre os estudos mostra que não há uma presença muito forte da "Relação Custo/Volume/Lucro" nos planos de ensino das universidades brasileiras, diferentemente nas universidades norte-americanas, e nas de Santa Catarina. O tópico "Conceitos e terminologia de custos" é muito presente no atual estudo e no de Splitter et al. (2014). No entanto, nos estudos de Raupp et al. (2009a) e Raupp et al. (2009b), esse tópico não apresenta muitas menções. O conteúdo "Ponto de equilíbrio" está muito presente tanto no atual estudo como no de Raupp et al. (2009b). Já não se percebe tanto no estudo de Splitter et al. (2014).

Portanto, percebem-se algumas semelhanças e diferenças entre o presente estudo e os outros dois. Em um âmbito geral, não se pode afirmar total homogeneidade ou total heterogeneidade entre os estudos, porém percebe-se maior similaridade do presente estudo com os resultados de Raupp et al. (2009a) e Raupp et al. (2009b).

\subsection{Metodologias de ensino citadas nos planos de ensino}

Treze (13) planos de ensino apresentavam o componente "Estratégias de ensino" em sua estrutura; portanto, eles foram analisados, e constatou-se que as metodologias de ensino utilizadas em todas as disciplinas são "Aulas Expositivas", "Exercícios em sala de aula" e "Atividades para casa".

O "Estudo de caso" aparece em 8 dos 13 planos de ensino, ou seja, $61 \%$. Já o "Trabalho em grupo" e o "Trabalho individual" foram mencionados em $61 \%$ e $54 \%$ dos planos, respectivamente. Os métodos menos utilizados são "Debates" e "Seminários", 
Contabilidade de Custos nas Universidades Brasileiras: uma análise dos planos de ensino nos cursos de Administração

Diego Sampaio Vasconcelos Ramalho Lima, Maria Naiula Monteiro Pessoa, Sandra Maria dos Santos, Augusto Cézar de Aquino Cabral, Clayton Robson Moreira da Silva

com 38\% de menções, ou seja, 5 dos 13 planos. Todas as metodologias de ensino mencionadas estão apresentadas na Tabela 5.

Tabela 5: Métodos de ensino utilizados

\begin{tabular}{|c|c|c|}
\hline Estratégia de ensino & Menções & $\%$ \\
\hline Aulas Expositivas & 13 & 100 \\
\hline Exercícios em sala de aula & 13 & 100 \\
\hline Atividades para casa & 13 & 100 \\
\hline Estudo de caso & 8 & 61,54 \\
\hline Trabalho em grupo & 7 & 53,84 \\
\hline Trabalho individuais & 6 & 46,15 \\
\hline Debates & 5 & 38,46 \\
\hline Seminários & 5 & 38,46 \\
\hline
\end{tabular}

Fonte: Elaborado pelos autores.

Nos estudos de Raupp et al. (2009a) e Raupp et al. (2009b), as "Aulas expositivas" correspondem à metodologia mais utilizada dentre as instituições. Os "Exercícios" ocupam o segundo lugar. Os "Estudos de caso" vêm logo depois e, por fim, encontram-se os "Trabalhos em equipe". Os "Seminários/apresentações", "Trabalhos individuais" e os "Debates/discussão em sala de aula" não ocuparam posição de destaque.

Já no estudo de Splitter et al. (2014), as "Aulas expositivas" e "Atividades para casa" correspondem às metodologias mais utilizadas dentre as universidades norteamericanas estudadas, sendo apresentadas em 100\% da amostra. Em seguida, "Atividades em aula", "Quizzes", "Trabalhos em grupo" e "Estudo de caso" apareceram em mais de $50 \%$ da amostra. Por fim, 12,5\% apresentaram "Revisões semanais" e "Seminários".

Portanto, a análise das metodologias mostra que há certa homogeneidade quanto ao atual estudo e os outros dois, tanto o brasileiro quanto o norteamericano. Pode-se observar que o Brasil não está muito distante da realidade norteamericana com relação ao método de ensino da disciplina de contabilidade de custos. 
Contabilidade de Custos nas Universidades Brasileiras: uma análise dos planos de ensino nos cursos de Administração

Diego Sampaio Vasconcelos Ramalho Lima, Maria Naiula Monteiro Pessoa, Sandra Maria dos Santos, Augusto Cézar de Aquino Cabral, Clayton Robson Moreira da Silva

\subsection{Técnicas de avaliação citadas nos planos de ensino}

$\mathrm{Na}$ análise dos métodos empregados para avaliação, foram verificados quais os instrumentos utilizados para a composição das notas. Na estrutura dos planos, 14 dos 34 apresentavam as técnicas avaliativas utilizadas. Analisando-as, percebe-se que, em 100\% das disciplinas, as "Provas parciais" são utilizadas, como mostra a Tabela 6.

Tabela 6: Técnicas avaliativas identificadas

\begin{tabular}{|c|c|c|}
\hline Métodos & Menções & $\%$ \\
\hline Provas parciais & 14 & 100 \\
\hline Exercícios & 11 & 78,57 \\
\hline Assiduidade & 11 & 78,57 \\
\hline Trabalho & 8 & 57,14 \\
\hline Estudos de caso & 3 & 21,43 \\
\hline Seminários & 2 & 14,28 \\
\hline
\end{tabular}

Fonte: Elaborado pelos autores.

Outras técnicas muito mencionadas foram: os "Exercícios", a "Assiduidade" e o "Trabalho", com $78 \%$ para as duas primeiras e $57 \%$ para a última. Por fim, as técnicas menos utilizadas são os "Estudos de caso" e "Seminários", com 21\% e 14\%, respectivamente.

No que concerne aos estudos de Raupp et al. (2009a) e Raupp et al. (2009b), a "Prova" é o único método presente em todos os planos que citam o sistema de avaliação, seguido pelos "Trabalhos" e "Exercícios", mencionados $82 \%$ e 45\%, respectivamente.

Já no estudo de Splitter et al. (2014), a duas formas de avaliação usadas em todos os planos de ensino foram "Prova final" e "Prova I", que corresponde à primeira prova. Os itens "Participação/presença" e "Atividades para casa" foram apresentados em $75 \%$ e $63 \%$ dos planos de ensino, respectivamente.

Portanto, conclui-se que, na análise comparativa entre os estudos, há forte homogeneidade quanto às técnicas avaliativas utilizadas nas disciplinas de custos. Nos 
Contabilidade de Custos nas Universidades Brasileiras: uma análise dos planos de ensino nos cursos de Administração

Diego Sampaio Vasconcelos Ramalho Lima, Maria Naiula Monteiro Pessoa, Sandra Maria dos Santos, Augusto Cézar de Aquino Cabral, Clayton Robson Moreira da Silva

três estudos, a "Prova" é a técnica mais utilizada. No topo do ranking, estão também os "Exercícios" e a "Presença".

\subsection{Bibliografias citadas nos planos de ensino}

O levantamento das principais bibliografias utilizadas nos planos de ensino constatou que existe uma quantidade muito grande de referências. Ao todo, foram coletadas 70 bibliografias; no entanto, como muitas eram mencionadas apenas uma, duas ou três vezes, apresenta-se o resultado a partir de quatro menções.

A análise das bibliografias utilizadas mostra que os planos de ensino trazem muitas opções de bibliografias. Cada plano tinha em torno de 8 bibliografias, divididas em básicas e complementares. O livro mais utilizado pelas Universidades é "Contabilidade de Custos", do autor Eliseu Martins, presente em $88 \%$ dos planos. Ele não foi mencionado apenas por 4 planos de ensino, estando presente, portanto, em 30 dos 34. Posteriormente, pôde-se verificar que não há forte menção de outra bibliografia. A bibliografia que aparece logo em seguida tem apenas 32\% de menção, que foi "Curso de contabilidade de custos", dos autores George Sebastião Guerra Leone e Rodrigo Jose Guerra Leone, conforme Tabela 7. 
Contabilidade de Custos nas Universidades Brasileiras: uma análise dos planos de ensino nos cursos de Administração

Diego Sampaio Vasconcelos Ramalho Lima, Maria Naiula Monteiro Pessoa, Sandra Maria dos Santos, Augusto Cézar de Aquino Cabral, Clayton Robson Moreira da Silva

Tabela 7: Principais bibliografias utilizadas nos planos de ensino

\begin{tabular}{|c|c|c|}
\hline Referências & Menções & $\%$ \\
\hline MARTINS, Eliseu. Contabilidade de Custos. 10. Ed., São Paulo: Atlas, 2010. & 30 & 88,23 \\
\hline $\begin{array}{l}\text { LEONE, George Sebastião Guerra; LEONE, Rodrigo José Guerra. Curso de } \\
\text { contabilidade de custos. 4. ed. São Paulo: Atlas, } 2010 .\end{array}$ & 11 & 32,35 \\
\hline $\begin{array}{c}\text { BRUNI, Adriano L.; FAMA, Rubens. Gestão de custos e formação de preços. } \\
\text { 5. ed. , São Paulo: Atlas, } 2008 .\end{array}$ & 9 & 26,47 \\
\hline $\begin{array}{c}\text { CREPALDI, Silvio Aparecido. Curso básico de contabilidade: resumo da } \\
\text { teoria. 5. ed. São Paulo: Atlas, } 2010 .\end{array}$ & 8 & 23,53 \\
\hline $\begin{array}{c}\text { HORNGREN, Charles T.; DATAR, Srikant M.; FOSTER, George. } \\
\text { Contabilidade de custos: uma abordagem gerencial. 11. ed. São Paulo: } \\
\text { Pearson Prentice Hall, 2004. }\end{array}$ & 7 & 20,59 \\
\hline $\begin{array}{l}\text { MAHER, Michael. Contabilidade de custos - criando valor para a } \\
\text { administração. 5. ed., São Paulo: Atlas, } 2001 .\end{array}$ & 7 & 20,59 \\
\hline $\begin{array}{c}\text { GARRISON, Ray H.; NOREEN, Eric W.; BREWER, Peter C. Contabilidade } \\
\text { gerencial. 11. ed. Rio de Janeiro: LTC, } 2007 .\end{array}$ & 6 & 17,64 \\
\hline IUDICIBUS, Sérgio de. Análise de Custos. São Paulo: Atlas, 2013. & 6 & 17,64 \\
\hline $\begin{array}{l}\text { OLIVEIRA, Luís Martins e PEREZ JUNIOR, José Hernandes. Contabilidade } \\
\text { de custos para não contadores. } 4 \text { ed. São Paulo: Atlas, } 2009 .\end{array}$ & 6 & 17,64 \\
\hline $\begin{array}{l}\text { ATKINSON, Anthony A.; BANKER, Rajiv. D.; KAPLAN, Robert; S. YOUNG, } \\
\text { S. M. Contabilidade Gerencial. São Paulo: Atlas, } 2000 .\end{array}$ & 5 & 14,70 \\
\hline $\begin{array}{c}\text { BORNIA, Antonio Cezar. Análise Gerencial de Custos: Aplicação em } \\
\text { Empresas Modernas. São Paulo: Atlas, 2009. }\end{array}$ & 5 & 14,70 \\
\hline $\begin{array}{l}\text { NAKAGAWA, Masayuki. Gestão Estratégica de Custos. São Paulo: Atlas, } \\
\qquad 2012 .\end{array}$ & 5 & 14,70 \\
\hline $\begin{array}{l}\text { BACKER, Morton e JACOBSEN, Lyle E. Contabilidade de custos. 3. ed. São } \\
\text { Paulo: McGraw-Hill, } 1980 .\end{array}$ & 4 & 11,76 \\
\hline $\begin{array}{c}\text { BELKE, Rolando; BERTÓ, Dalvio. Gestão de Custos. 2. ed. São Paulo: } \\
\text { Saraiva, } 2012 .\end{array}$ & 4 & 11,76 \\
\hline DUTRA, Rene Gomes. Custos: uma abordagem prática. Atlas. & 4 & 11,76 \\
\hline $\begin{array}{c}\text { LEONE, George Sebastião Guerra. Custos, planejamento, implantação e } \\
\text { controle. } 3 \text { ed. São Paulo, Atlas, } 2000 .\end{array}$ & 4 & 11,76 \\
\hline $\begin{array}{c}\text { VANDERBECK, Edward J.; NAGY, Charles. F. Contabilidade de custos. } 11 . \\
\text { ed. São Paulo: Thomson Learning, } 2003 .\end{array}$ & 4 & 11,76 \\
\hline
\end{tabular}

Fonte: Elaborado pelos autores.

Nos estudos de Raupp et al. (2009a) e Raupp (2009b), também é possível perceber uma discrepância proeminente no que se refere ao ranking de ocorrências. $O$ 
Contabilidade de Custos nas Universidades Brasileiras: uma análise dos planos de ensino nos cursos de Administração

Diego Sampaio Vasconcelos Ramalho Lima, Maria Naiula Monteiro Pessoa, Sandra Maria dos Santos, Augusto Cézar de Aquino Cabral, Clayton Robson Moreira da Silva

livro mais citado é "Contabilidade de Custos", de Eliseu Martins (2010), com 22 ocorrências dentre 26 analisadas, seguido da obra "Análise gerencial de custos: aplicação em empresas modernas", de Antônio Cezar Bornia (2009), indicado por 8 planos. Uma quantidade muito grande de obras é citada nos planos de ensino.

Já no estudo de Splitter et al. (2014), foi verificado que apenas um plano de ensino utiliza dois livros, e o restante adota apenas um livro. Os resultados demonstram que Horngren, Datar, Foster, Rajan e Ittner (2008), "Cost Accounting: A Managerial Emphasis" representa 7 dos 17 livros apresentados nos planos de ensino. O primeiro autor ainda aparece em outra obra: "Introduction to Management Accounting". Os autores Garrison, Noreen e Brewer (2010) aparecem em segundo lugar, com 3 menções em planos de ensino, com o livro "Managerial Accounting" e mais um com a obra "Introduction to Managerial Accounting".

Portanto, na análise entre o presente estudo e o estudo nas Instituições de Santa Catarina, percebe-se semelhança, visto que ambos apresentam vasta gama de bibliografias nos planos de ensino, e há predominância forte do livro de Eliseu Martins "Contabilidade de Custos". Por outro lado, a comparação com o estudo norteamericano não mostra semelhança, já que ele apresenta poucas bibliografias e utiliza livros diferentes dos utilizados pelo presente estudo.

Por meio da análise dos resultados, portanto, percebe-se que cada estudo possui suas peculiaridades, não se podendo dizer que há plena similaridade entre o presente estudo e os de Splitter et al. (2014), Raupp et al. (2009a) e Raupp et al. (2009b). No entanto, pode-se afirmar uma maior proximidade com os resultados de Raupp et al. (2009a) e Raupp (2009b).

\section{CONSIDERAÇÕES FINAIS}

Visando contribuir com o ensino da disciplina de custos, o presente estudo foi desenvolvido com o propósito de identificar o perfil da disciplina de custos no ensino de graduação em Administração nas universidades brasileiras, por meio de análise na 
Contabilidade de Custos nas Universidades Brasileiras: uma análise dos planos de ensino nos cursos de Administração

Diego Sampaio Vasconcelos Ramalho Lima, Maria Naiula Monteiro Pessoa, Sandra Maria dos Santos, Augusto Cézar de Aquino Cabral, Clayton Robson Moreira da Silva

estrutura dos seus planos de ensino e identificação dos elementos - conteúdos abordados, referências utilizadas, métodos de ensino e formas avaliativas empregadas. Ao mesmo tempo, buscou-se comparar as semelhanças e particularidades dos resultados aqui alcançados com os obtidos em estudos anteriores.

Quanto ao perfil identificado, percebe-se que há grande variação quanto à nomenclatura utilizada para identificar a disciplina de custos, sendo a disciplina, em sua maioria, chamada de: "Contabilidade de Custos", "Contabilidade Gerencial" e "Gestão de Custos". Ao que se refere à estrutura dos planos de ensino analisados, foi possível observar que os elementos presentes em todos os planos de ensino foram: "Disciplina" e "Bibliografia". Os itens "Ementa", "Carga horária", "Objetivos" e "Conteúdo" tiveram grande representatividade. Por outro lado, o componente "Cronograma" não costuma ser mencionado na estrutura dos planos.

No que tange aos conteúdos expostos nos planos de ensino em análise, identificou-se certa homogeneidade quanto às matérias lecionadas na disciplina, que são, dentre outras, "Conceitos e Terminologia de Custos", "Classificação dos custos e despesas", "Custeio variável" e "Custeio por absorção". Sobre os métodos de ensino empregados, constatou-se que as "Aulas Expositivas", os "Exercícios em sala de aula" e as "Atividades para casa" são técnicas utilizadas em todas as disciplinas.

Constatou-se ainda que, quanto aos métodos avaliativos, em todas as disciplinas, as "Provas parciais" são utilizadas. Outras técnicas muito mencionadas foram: os "Exercícios", a "Assiduidade" e "Trabalho". As técnicas menos utilizadas são os "Estudos de caso" e "Seminários". Quanto às bibliografias, verificou-se uma pluralidade de referências distintas, predominando o autor Martins (2010), com seu livro "Contabilidade de Custos" como o mais citado.

Por meio da comparação entre o presente estudo e estudos anteriores, percebese que cada um possui suas peculiaridades, não se podendo dizer que há plena similaridade entre os resultados aqui obtidos e os resultantes da pesquisa realizada em instituições americanas e estudo feito nas universidades de Santa Catarina - Brasil. No 
Contabilidade de Custos nas Universidades Brasileiras: uma análise dos planos de ensino nos cursos de Administração

Diego Sampaio Vasconcelos Ramalho Lima, Maria Naiula Monteiro Pessoa, Sandra Maria dos Santos, Augusto Cézar de Aquino Cabral, Clayton Robson Moreira da Silva

entanto, pode-se afirmar existir maior proximidade com os resultados do estudo catarinense.

É importante destacar que os resultados provenientes deste estudo estão relacionados com as universidades analisadas e seus planos de ensino, não podendo, dessa forma, ser generalizados. Por isso, a questão de pesquisa de identificar o perfil da disciplina de custos no ensino de graduação em Administração nas universidades brasileiras só pôde ser respondida em parte, restringindo-se às universidades devidamente representadas por seus planos de ensino. Além disso, a análise comparativa que fora desenvolvida serve apenas como embasamento e alinhamento entre os estudos, a fim de voltar a discussões futuras.

Por fim, espera-se que este trabalho contribua para uma ampliação dos estudos que envolvem a identificação da qualidade profissional passada pelas instituições de ensino aos seus alunos por meio da discussão dos conteúdos programáticos, ementas curriculares, técnicas de pesquisas e, assim, expor a necessidade de adequação das universidades às novas necessidades empresariais.

Algumas sugestões de pesquisas futuras são as comparações e análises das disciplinas entre as Instituições de ensino públicas e privadas ou entre as regiões do país. Outra sugestão seria realizar pesquisas com os docentes e coordenadores dos cursos, a fim de investigar o processo de ensino das disciplinas. A temática é bem ampla e ainda será muito discutida em futuras pesquisas.

\section{REFERÊNCIAS}

ANDRADE, M. M. (2008). Como preparar trabalhos para cursos de pós-graduação: noções práticas. São Paulo, Atlas, 168 p.

BRASIL. CNE. Conselho Nacional de Educação. Resolução $n^{\circ}$ 4, de 13 de julho de 2005. Institui as Diretrizes Curriculares Nacionais do Curso de Graduação em Administração, bacharelado, e dá outras providências. Disponível em: <http://portal.mec.gov.br/cne/arquivos/pdf/rces004_05.pdf>. Acesso em: 14/out/2015. 
Contabilidade de Custos nas Universidades Brasileiras: uma análise dos planos de ensino nos cursos de Administração

Diego Sampaio Vasconcelos Ramalho Lima, Maria Naiula Monteiro Pessoa, Sandra Maria dos Santos, Augusto Cézar de Aquino Cabral, Clayton Robson Moreira da Silva

CANOPF, L., FESTINALLI, R. C. e ICHIKAWA, E. Y. (2005). A expansão do ensino superior em administração no sudoeste do Paraná: reflexões introdutórias. Revista de Administração Contemporânea, 9(3): 79-97.

COVRE, M. L. M. (1991). A formação e a ideologia do administrador de empresas. São Paulo: Cortez, 202 p.

CREPALDI, S. A. (2010). Curso básico de contabilidade de custos. São Paulo, Atlas, $384 \mathrm{p}$.

DESLAURIERS, J. P. e KÉRISIT, M. (2008). O delineamento de pesquisa qualitativa. In: POUPART, J. et al. A pesquisa qualitativa: enfoques epistemológicos e metodológicos. Petrópolis, Vozes, p. 127-153.

FOLHA de São Paulo. (2015). Ranking Universitário Folha 2015. Ranking de universidades. Brasil. Disponível em: <http://ruf.folha.uol.com.br/2015/>. Acesso em: 14/out/2015.

GIL, A. C. (2011). Didática do ensino superior. São Paulo, Atlas, 286 p.

GODOY, A. S., SILVA, H. A. e NAKAMURA, W. T. (2004). Análise dos programas de ensino da área de custos no currículo dos cursos de graduação em administração de empresas. Revista Eletrônica de Administração, 10(4): 1-18.

HARADA, R. S. (2005). O ensino de contabilidade no curso de administração de empresas: a percepção dos docentes da disciplina de contabilidade geral em faculdades localizadas no município de São Paulo. São Paulo, SP. (Dissertação de mestrado). Fundação Escola de Comércio Álvares Penteado, 109 f.

HART, D. L. e WANG, K. (2016). Teaching accounting effectively: an examination of accounting students and faculty perceptions. Academy of Educational Leadership Journal, 20(1): 93-107.

HOPER, T. (2012). Making accounting degrees fit for a university. Critical Perspectives on Accounting, 24(1): 127-135.

KONT, K. R. (2013). Cost accounting and scientific management in libraries: a historical review. Journal of Management History, 19(2): 225-240.

LEAL, R. B. (2005). Planejamento de ensino: peculiaridades significativas. Revista Iberoamericana de Educación, 37(3): 1-6. 
Contabilidade de Custos nas Universidades Brasileiras: uma análise dos planos de ensino nos

cursos de Administração

Diego Sampaio Vasconcelos Ramalho Lima, Maria Naiula Monteiro Pessoa, Sandra Maria dos Santos, Augusto Cézar de Aquino Cabral, Clayton Robson Moreira da Silva

LOPES, P. (2002). Reflexões sobre as bases da formação do administrador profissional no ensino de graduação. In: Encontro Anual da ANPAD, 26, Salvador. Anais... Salvador, ANPAD, p. 1-16.

MARCONI, M. A. e LAKATOS, E. M. (2008). Técnicas de pesquisa. São Paulo, Atlas, $289 \mathrm{p}$.

MEGLIORINI, E. (2012). Custos: análise e gestão. São Paulo, Pearson Prentice Hall, $304 \mathrm{p}$.

NICOLINI, A. (2003). Qual será o futuro das fábricas de administradores? Revista de Administração de Empresas - RAE, 43(2): 44-54.

PADILHA, P. R. (2002). Planejamento dialógico: como construir o projeto políticopedagógico da escola. São Paulo, Cortez, 160 p.

PILETTI, C. (2010). Didática geral. São Paulo, Ática, 258 p.

RAUPP, F. M., AMBONI, N., CUNHA, D. R., DUARTE, J. F. e AGOSTINETO, R. C. (2009a). O ensino de Contabilidade geral e Contabilidade de custos nos cursos de graduação em administração do estado de Santa Catarina. Revista de Negócios, 14(2): 71-88.

RAUPP, F. M., AMBONI, N., CUNHA, D. R., DUARTE, J. F. e AGOSTINETO, R. C. (2009b). O ensino de contabilidade de custos nos cursos de graduação em administração do Estado de Santa Catarina. ABCustos - Associação Brasileira de Custos, 4(2):97-117.

RICHARDSON, R. J. (2008). Pesquisa social: métodos e técnicas. São Paulo, Atlas, $334 \mathrm{p}$.

SAMPIERI, R. H., COLLADO, C. F. e LUCIO, P. B. (2013). Metodologia de pesquisa. São Paulo, Penso, 624 p.

SOMBRA, A. M. M., PESSOA, M. N. M., CABRAL, A. C. A., SANTOS, S. M. e SILVA, J. D. (2016). Perfil da disciplina de contabilidade de custos nas universidades brasileiras: uma análise nos cursos de graduação em ciências contábeis. Revista de Gestão Organizacional, 9(1):66-82.

SPLITTER, K., FERREIRA, A. F., BORGERT, A., BORBA, J. A. e VAN BELLEN, H. M. (2014). Contabilidade de custos nas universidades norte-americanas: o perfil da disciplina nos cursos de graduação. Custos e @gronegócio online, 10(2): 251-271. 
Contabilidade de Custos nas Universidades Brasileiras: uma análise dos planos de ensino nos cursos de Administração

Diego Sampaio Vasconcelos Ramalho Lima, Maria Naiula Monteiro Pessoa, Sandra Maria dos Santos, Augusto Cézar de Aquino Cabral, Clayton Robson Moreira da Silva

TCHEOU, E. (2002). Avaliação do ensino de contabilidade nos cursos de administração de empresas na cidade de São Paulo. São Paulo, SP. (Dissertação de mestrado). Fundação Escola de Comércio Álvares Penteado, 203 f.

XAVIER FILHO, J. L. J., DIAS, S. A., FRANÇA, F. M., SILVA, A. C. B. e VASCONCELOS, A. F. (2015). A importância dos conhecimentos contábeis para discentes em Administração: uma análise a partir de influentes de julgamento. Gestão, Finanças e Contabilidade, 5(1): 77-104.

Data de Submissão: 04/10/2016

Data de Aceite: 29/12/2016 